Homology, Homotopy and Applications, vol.6(1), 2004, pp.341-350

\title{
NOTE ON THE RATIONAL COHOMOLOGY OF THE FUNCTION SPACE OF BASED MAPS
}

\author{
YASUSUKE KOTANI \\ (communicated by Hvedri Inassaridze)
}

\begin{abstract}
In this paper, for a formal, path connected, finite-dimensional CW-complex $X$ of finite type and a $q$-connected space $Y$ of finite type with $q \geqslant \operatorname{dim} X$, we determine the necessary and sufficient condition for the rational cohomology algebra $H^{*}\left(\mathcal{F}_{*}(X, Y) ; \mathbb{Q}\right)$ of the function space $\mathcal{F}_{*}(X, Y)$ of based maps to be free.
\end{abstract}

\section{Introduction}

Let $\mathcal{F}(X, Y)$ and $\mathcal{F}_{*}(X, Y)$ be function spaces of free maps and based maps from a space $X$ to a space $Y$ respectively. Then $\mathcal{F}(X, Y)$ and $\mathcal{F}_{*}(X, Y)$ are path connected if $X$ is a path connected, finite-dimensional CW-complex of finite type and $Y$ is a $q$-connected space with $q \geqslant \operatorname{dim} X$.

A commutative graded algebra $A=\left\{A^{p}\right\}_{p \geqslant 0}$ satisfying $A^{0}=\mathbb{Q}$ is said to be free if $A$ is isomorphic to a free commutative graded algebra $\wedge V$ on a graded vector space $V$.

A commutative cochain algebra $(A, d)$ satisfying $H^{0}(A)=\mathbb{Q}$ is said to be formal if $(A, d)$ and $(H(A), 0)$ are connected by a chain of quasi-isomorphisms. A path connected space $X$ is said to be formal if the commutative cochain algebra $A_{\mathrm{PL}}(X)$ of rational polynomial differential forms on $X$ is formal.

It is known that, for an arbitrary $n$-connected space $Y$ with $n \geqslant 1$, the rational cohomology algebra

$$
H^{*}\left(\Omega^{n} Y ; \mathbb{Q}\right)=H^{*}\left(\mathcal{F}_{*}\left(S^{n}, Y\right) ; \mathbb{Q}\right)
$$

of the $n$-fold loop space $\Omega^{n} Y$ of $Y$ is free, and that spheres $S^{n}$ are formal.

In this paper, for a formal, path connected, finite-dimensional CW-complex $X$ of finite type and a $q$-connected space $Y$ of finite type with $q \geqslant \operatorname{dim} X$, we consider the condition for the rational cohomology algebra $H^{*}\left(\mathcal{F}_{*}(X, Y) ; \mathbb{Q}\right)$ of the function space $\mathcal{F}_{*}(X, Y)$ of based maps to be free.

Let $H^{*}(X ; \mathbb{Q})=\left\{H^{p}(X ; \mathbb{Q})\right\}_{p \geqslant 0}$ be the rational cohomology algebra for a path connected space $X$ with the cup product

$$
\cup: H^{*}(X ; \mathbb{Q}) \otimes H^{*}(X ; \mathbb{Q}) \rightarrow H^{*}(X ; \mathbb{Q}) .
$$

Received October 23, 2003, revised July 12, 2004; published on August 6, 2004. 2000 Mathematics Subject Classification: Primary 55P62; Secondary 54C40.

Key words and phrases: rational cohomology, function spaces, free commutative graded algebras, formal spaces.

(C) 2004, Yasusuke KOTANI. Permission to copy for private use granted. 
Recall that the rational cup length $\operatorname{cup}(X ; \mathbb{Q})$ of $X$ is defined by

$$
\sup \left\{n \in \mathbb{Z} \mid f_{1} \cup \cdots \cup f_{n} \neq 0 \text { for } f_{1}, \ldots, f_{n} \in H^{+}(X ; \mathbb{Q})\right\},
$$

where $H^{+}(X ; \mathbb{Q})=\left\{H^{p}(X ; \mathbb{Q})\right\}_{p>0}$.

Let $(\wedge V, d)$ be a Sullivan algebra. Elements in $\wedge V$ of the form $v_{1} \wedge \cdots \wedge v_{k}$ for $v_{1}, \ldots, v_{k} \in V$ are said to have word length $k$. Then the differential $d$ decomposes uniquely as the sum

$$
d=d_{0}+d_{1}+d_{2}+\cdots
$$

of derivations $d_{i}$ raising the word length by $i$. (cf. [3, Section 12(a)]). Now, we define the differential length $\operatorname{dl}(\wedge V, d)$ of $(\wedge V, d)$ by the least integer $m$ such that $d_{m-1} \neq 0$. If $d_{i}=0$ for all $i \geqslant 0$, that is, $d=0$, we define $\operatorname{dl}(\wedge V, 0)=\infty$. We also define the differential length $\mathrm{dl}(Y)$ of a simply connected space $Y$ of finite type by that of a minimal Sullivan model for $Y$. Then we can establish

Theorem 1.1. The differential length of a simply connected space of finite type is independent of a choice of minimal Sullivan models. Thus it is a rational homotopy invariant.

Our main theorem is as follows.

Theorem 1.2. Let $X$ be a formal, path connected, finite-dimensional CW-complex of finite type and $Y$ a q-connected space of finite type with $q \geqslant \operatorname{dim} X$. Then $H^{*}\left(\mathcal{F}_{*}(X, Y) ; \mathbb{Q}\right)$ is free if and only if $\operatorname{cup}(X ; \mathbb{Q})<\operatorname{dl}(Y)$.

This paper is organized as follows. In Section 2, we recall the construction of a minimal Sullivan model for $\mathcal{F}(X, Y)$ due to E. H. Brown, Jr. and R. H. Szczarba [2, Thoerem 1.9]. Moreover, we describe a minimal Sullivan model for $\mathcal{F}_{*}(X, Y)$ is obtained by that for $\mathcal{F}(X, Y)$ using the evaluation fibration, which is established by K. Kuribayashi [4, Theorem 3.6]. The proofs of Theorems are given in Section 3 and 4 respectively. In Section 5, we give some examples.

The author would like to express his deepest gratitude to Professor K. Kuribayashi for permitting to use some results in [4, Section 3], and also to Professor T. Yamaguchi for helpful suggestions.

\section{Minimal Sullivan models for $\mathcal{F}(X, Y)$ and $\mathcal{F}_{*}(X, Y)$}

Let $X$ and $Y$ be as in Theorem 1.2. Then the construction of a minimal Sullivan model for $\mathcal{F}(X, Y)$ due to E. H. Brown, Jr. and R. H. Szczarba [2, Theorem 1.9] is described as follows.

Let $m_{Y}:(\wedge V, d) \stackrel{\simeq}{\longrightarrow} A_{\mathrm{PL}}(Y)$ be a minimal Sullivan model for $Y$. Let $H_{*}(X ; \mathbb{Q})=$ $\left\{H_{p}(X ; \mathbb{Q})\right\}_{p} \geqslant 0$ be the rational homology coalgebra for $X$ with the coproduct

$$
\Delta: H_{*}(X ; \mathbb{Q}) \rightarrow H_{*}(X ; \mathbb{Q}) \otimes H_{*}(X ; \mathbb{Q}) .
$$

Let $\wedge V \otimes H_{*}(X ; \mathbb{Q})$ be a graded vector space with grading $|v \otimes c|=|v|-|c|$ for $v \in \wedge V$ and $c \in H_{*}(X ; \mathbb{Q})$. Let $\wedge\left(\wedge V \otimes H_{*}(X ; \mathbb{Q})\right)$ be the free commutative graded 
algebra on $\wedge V \otimes H_{*}(X ; \mathbb{Q})$ with the differential $d \otimes$ id, and let $I$ be the ideal in $\wedge\left(\wedge V \otimes H_{*}(X ; \mathbb{Q})\right)$ generated by $1 \otimes 1-1$ and all elements of the form

$$
v^{\prime} \wedge v^{\prime \prime} \otimes c-\sum(-1)^{\left|v^{\prime \prime}\right|\left|c_{j}^{\prime}\right|}\left(v^{\prime} \otimes c_{j}^{\prime}\right) \wedge\left(v^{\prime \prime} \otimes c_{j}^{\prime \prime}\right)
$$

for $v^{\prime}, v^{\prime \prime} \in \wedge V$ and $c \in H_{*}(X ; \mathbb{Q})$ with $\Delta c=\sum c_{j}^{\prime} \otimes c_{j}^{\prime \prime}$. Then $(d \otimes$ id $)(I) \in I([\mathbf{2}$, Theorem 3.3]) and the composition map

$$
\rho: \wedge\left(V \otimes H_{*}(X ; \mathbb{Q})\right) \hookrightarrow \wedge\left(\wedge V \otimes H_{*}(X ; \mathbb{Q})\right) \rightarrow \wedge\left(\wedge V \otimes H_{*}(X ; \mathbb{Q})\right) / I
$$

is an isomorphism of graded algebras $([\mathbf{2}$, Theorem 3.3]). Let $\delta$ be the differential on $\wedge\left(V \otimes H_{*}(X ; \mathbb{Q})\right)$ given by $\delta=\rho^{-1}(d \otimes$ id $) \rho$. Then, by [2, Theorem 1.9], $\mathcal{F}(X, Y)$ has a minimal Sullivan model of the form

$$
\left(\wedge\left(V \otimes H_{*}(X ; \mathbb{Q})\right), \delta\right) .
$$

Next, let us consider the evaluation fibration

$$
\mathcal{F}_{*}(X, Y) \rightarrow \mathcal{F}(X, Y) \stackrel{e v_{*}}{\longrightarrow} Y,
$$

where $e v_{*}$ is the evaluation map at the basepoint of $X$. Let $i:(\wedge V, d) \hookrightarrow(\wedge(V \otimes$ $\left.\left.H_{*}(X ; \mathbb{Q})\right), \delta\right)$ be the inclusion map defined by $i(v)=v \otimes 1$ for $v \in V$. From the consideration in $[\mathbf{4}$, Section 3], we have a commutative diagram

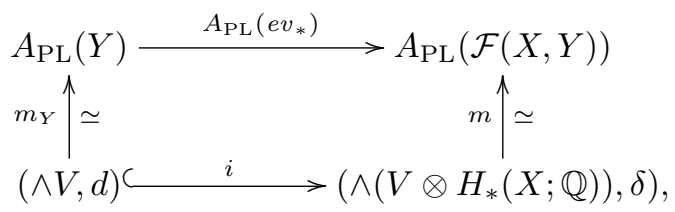

where $m:\left(\wedge\left(V \otimes H_{*}(X ; \mathbb{Q})\right), \delta\right) \stackrel{\simeq}{\longrightarrow} A_{\mathrm{PL}}(\mathcal{F}(X, Y))$ is a minimal Sullivan model for $\mathcal{F}(X, Y)$ described above. Thus the inclusion map $i$ is viewed as a model for the evaluation map $e v_{*}$.

Let $J$ be an ideal of $\wedge\left(V \otimes H_{*}(X ; \mathbb{Q})\right)$ generated by $v \otimes 1$ for $v \in V$. Let $\bar{\delta}$ be the differential on $\wedge\left(V \otimes H_{*}(X ; \mathbb{Q})\right) / J$ induced from $\delta$ on $\wedge\left(V \otimes H_{*}(X ; \mathbb{Q})\right)$. Then, by $\left[\mathbf{3}\right.$, Proposition 15.5] and $\left[\mathbf{4}\right.$, Theorem 3.6], $\mathcal{F}_{*}(X, Y)$ has a minimal Sullivan model of the form

$$
\left(\wedge\left(V \otimes H_{*}(X ; \mathbb{Q})\right) / J, \bar{\delta}\right)=\left(\wedge\left(V \otimes H_{+}(X ; \mathbb{Q})\right), \bar{\delta}\right),
$$

where $H_{+}(X ; \mathbb{Q})=\left\{H_{p}(X ; \mathbb{Q})\right\}_{p>0}$.

\section{Proof of Theorem 1.1}

It is known that minimal Sullivan models for a simply connected space of finite type are all isomorphic, and that the isomorphism class of a minimal Sullivan model for a simply connected space of finite type is a rational homotopy invariant. Hence, for the proof of Theorem 1.1, it is sufficient to prove the following.

Proposition 3.1. Let $(\wedge V, d)$ and $\left(\wedge V^{\prime}, d^{\prime}\right)$ be isomorphic Sullivan algebras. Then $\operatorname{dl}(\wedge V, d)=\operatorname{dl}\left(\wedge V^{\prime}, d^{\prime}\right)$. 
Proof. Let $f:(\wedge V, d) \stackrel{\cong}{\longrightarrow}\left(\wedge V^{\prime}, d^{\prime}\right)$ be an isomorphism of differential graded algebras.

First, suppose that $\operatorname{dl}\left(\wedge V^{\prime}, d^{\prime}\right)=\infty$, that is, $d^{\prime}=0$. Then, since $f d=d^{\prime} f=0$ and $f$ is an isomorphism, we have $d=0$. Thus $\operatorname{dl}(\wedge V, d)=\infty$.

Next, suppose that $\operatorname{dl}\left(\wedge V^{\prime}, d^{\prime}\right)=m<\infty$, that is, $d_{i}^{\prime}=0$ for $0 \leqslant i<m-1$ and $d_{m-1}^{\prime} \neq 0$. Then, since $f$ is an isomorphism, for an arbitrary element $v \in V$, there exists an element $v^{\prime} \in V^{\prime}$ such that

$$
f(v)=v^{\prime}+\text { (higher terms). }
$$

Now, assume that $d v$ has terms of the form $v_{1} \wedge \cdots \wedge v_{k}$ for $v_{1}, \ldots, v_{k} \in V$ and $k \leqslant m-1$. Then $f(d v)$ has terms of the form

$$
f\left(v_{1} \wedge \cdots \wedge v_{k}\right)=f\left(v_{1}\right) \wedge \cdots \wedge f\left(v_{k}\right)=v_{1}^{\prime} \wedge \cdots \wedge v_{k}^{\prime}+\text { (higher terms) }
$$

for $v_{1}^{\prime}, \ldots, v_{k}^{\prime} \in V^{\prime}$ and $k \leqslant m-1$. However, $d^{\prime} f(v)=f(d v)$ has no such terms because $d_{i}^{\prime}=0$ for $0 \leqslant i<m-1$. It is a contradiction. Hence we have $d_{i}=0$ for $0 \leqslant i<m-1$ since $d$ is a derivation. So we get the inequality $\operatorname{dl}(\wedge V, d) \geqslant$ $\operatorname{dl}\left(\wedge V^{\prime}, d^{\prime}\right)$. Since $f^{-1}$ is also an isomorphism, we get the inverse inequality. Thus $\operatorname{dl}(\wedge V, d)=\operatorname{dl}\left(\wedge V^{\prime}, d^{\prime}\right)=m$.

\section{Proof of Theorem $\mathbf{1 . 2}$}

Let $X$ and $Y$ be as in Theorem 1.2. Let $(\wedge V, d)$ be a minimal Sullivan model for $Y$ and $H_{*}(X ; \mathbb{Q})$ the rational homology coalgebra for $X$. Then, as described in Section $2, \mathcal{F}_{*}(X, Y)$ has a minimal Sullivan model of the form

$$
\left(\wedge\left(V \otimes H_{+}(X ; \mathbb{Q})\right), \bar{\delta}\right),
$$

where $\bar{\delta}$ is induced from $\delta=\rho^{-1}(d \otimes$ id $) \rho$ on $\wedge\left(V \otimes H_{*}(X ; \mathbb{Q})\right)$ by reducing elements contained in the ideal $J$ generated by $v \otimes 1$ for $v \in V$.

It is easy to see that $H^{*}\left(\mathcal{F}_{*}(X, Y) ; \mathbb{Q}\right) \cong H\left(\wedge\left(V \otimes H_{+}(X ; \mathbb{Q})\right), \bar{\delta}\right)$ is free if and only if $\bar{\delta}=0$, and that $\bar{\delta}=0$ if and only if $\delta\left(\wedge\left(V \otimes H_{+}(X ; \mathbb{Q})\right)\right) \in J$. Hence, for the proof of Theorem 1.2, it is sufficient to prove the following.

Proposition 4.1. (1). If $\operatorname{cup}(X ; \mathbb{Q})<\operatorname{dl}(Y)$, then $\delta\left(\wedge\left(V \otimes H_{+}(X ; \mathbb{Q})\right)\right) \in J$ or equivalently $\bar{\delta}=0$.

(2). If $\operatorname{cup}(X ; \mathbb{Q}) \geqslant \mathrm{dl}(Y)$, then $\delta\left(\wedge\left(V \otimes H_{+}(X ; \mathbb{Q})\right)\right) \notin J$.

Thus we need to explain the differential $\delta$ in detail. Let $\Delta$ be the coproduct on $H_{*}(X ; \mathbb{Q})$. Then the reduced coproduct

$$
\bar{\Delta}: H_{+}(X ; \mathbb{Q}) \rightarrow H_{+}(X ; \mathbb{Q}) \otimes H_{+}(X ; \mathbb{Q})
$$

is defined by $\bar{\Delta} c=\Delta c-c \otimes 1-1 \otimes c$ for $c \in H_{+}(X ; \mathbb{Q})$. Moreover, the $k$-th coproduct $\Delta^{(k)}$ and the $k$-th reduced coproduct $\bar{\Delta}^{(k)}$ are defined inductively by $\Delta^{(0)}=\bar{\Delta}^{(0)}=$ id, $\Delta^{(1)}=\Delta, \bar{\Delta}^{(1)}=\bar{\Delta}$ and

$$
\begin{aligned}
& \Delta^{(k)}=(\Delta \otimes \mathrm{id} \otimes \cdots \otimes \mathrm{id}) \circ \Delta^{(k-1)}: H_{*}(X ; \mathbb{Q}) \rightarrow H_{*}(X ; \mathbb{Q})^{\otimes k+1}, \\
& \bar{\Delta}^{(k)}=(\bar{\Delta} \otimes \mathrm{id} \otimes \cdots \otimes \mathrm{id}) \circ \bar{\Delta}^{(k-1)}: H_{+}(X ; \mathbb{Q}) \rightarrow H_{+}(X ; \mathbb{Q})^{\otimes k+1},
\end{aligned}
$$


where $H^{\otimes k+1}$ denotes the $(k+1)$-times tensor product of $H$.

Let $H^{*}(X ; \mathbb{Q})$ be the rational cohomology algebra for $X$ with the cup product $\cup$. Since $X$ is of finite type, $H^{*}(X ; \mathbb{Q})$ with $\cup$ and $H_{*}(X ; \mathbb{Q})$ with $\Delta$ are dual each other. Hence we have immediately

Lemma 4.2. If $\operatorname{cup}(X ; \mathbb{Q})=n$, then $\bar{\Delta}^{(k-1)} \neq 0$ for $0<k \leqslant n$ and $\bar{\Delta}^{(k-1)}=0$ for all $k>n$.

Let $B_{H_{*}}=\left\{c_{0}=1, c_{1}, c_{2}, \ldots\right\}$ be a basis for $H_{*}(X ; \mathbb{Q})$ with $0<\left|c_{1}\right| \leqslant\left|c_{2}\right| \leqslant \cdots$. Then, for an arbitrary element $c_{j} \in B_{H_{*}}$ and $k \geqslant 2$, we may denote

$$
\Delta^{(k-1)} c_{j}=\sum \mu_{j_{1}, \ldots, j_{k}} c_{j_{1}} \otimes \cdots \otimes c_{j_{k}}
$$

where $0 \neq \mu_{j_{1}, \ldots, j_{k}} \in \mathbb{Q}$ and $c_{j_{1}}, \ldots, c_{j_{k}} \in B_{H_{*}}$. By the definition of the reduced coproduct, we have immediately

Lemma 4.3. $\bar{\Delta}^{(k-1)} c_{j}=0$ if and only if there exists an integer $s$ such that $c_{j_{s}}=1$ in each term of $\Delta^{(k-1)} c_{j}$.

Moreover, since the cup product $U$ is associative and commutative, so is the coproduct $\Delta$, that is, $(\Delta \otimes$ id $) \Delta=(\mathrm{id} \otimes \Delta) \Delta$ and $\tau \Delta=\Delta$, where $\tau$ is defined by $\tau\left(c \otimes c^{\prime}\right)=(-1)^{|c|\left|c^{\prime}\right|} c^{\prime} \otimes c$. Hence we have immediately

Lemma 4.4. $\mu_{j_{1}, \ldots, j_{s}, j_{s+1}, \ldots, j_{k}}=(-1)^{\left|c_{j_{s}}\right|\left|c_{j_{s+1}}\right|} \mu_{j_{1}, \ldots, j_{s+1}, j_{s}, \ldots, j_{k}}$.

Let $B_{V}=\left\{v_{1}, v_{2}, \ldots\right\}$ be a basis for $V$ with $0<\left|v_{1}\right| \leqslant\left|v_{2}\right| \leqslant \cdots$. Then, if $d v_{i}=v_{i_{1}} \wedge \cdots \wedge v_{i_{k}}$ for $v_{i} \in B_{V}$ and $\Delta^{(k-1)} c_{j}=\sum \mu_{j_{1}, \ldots, j_{k}} c_{j_{1}} \otimes \cdots \otimes c_{j_{k}}$ for $c_{j} \in B_{H_{*}}$, we have

$$
\delta\left(v_{i} \otimes c_{j}\right)=\sum(-1)^{\varepsilon\left(i_{1}, j_{1} ; \ldots ; i_{k}, j_{k}\right)} \mu_{j_{1}, \ldots, j_{k}}\left(v_{i_{1}} \otimes c_{j_{1}}\right) \wedge \cdots \wedge\left(v_{i_{k}} \otimes c_{j_{k}}\right),
$$

where the $\operatorname{sign}(-1)^{\varepsilon\left(i_{1}, j_{1} ; \ldots ; i_{k}, j_{k}\right)}$ is determined by (2.1), that is,

$$
v_{i_{1}} \wedge \cdots \wedge v_{i_{k}} \otimes c_{j}=\sum(-1)^{\varepsilon\left(i_{1}, j_{1} ; \ldots ; i_{k}, j_{k}\right)} \mu_{j_{1}, \ldots, j_{k}}\left(v_{i_{1}} \otimes c_{j_{1}}\right) \wedge \cdots \wedge\left(v_{i_{k}} \otimes c_{j_{k}}\right)
$$

in the graded algebra $\wedge\left(\wedge V \otimes H_{*}(X ; \mathbb{Q})\right) / I$. More precisely, $\varepsilon\left(i_{1}, j_{1} ; \ldots ; i_{k}, j_{k}\right)$ is given by

Lemma 4.5. $\varepsilon\left(i_{1}, j_{1} ; \ldots ; i_{k}, j_{k}\right)=\sum_{l=1}^{k-1}\left(\left|v_{i_{l+1}}\right|+\cdots+\left|v_{i_{k}}\right|\right)\left|c_{j_{l}}\right|$

Proof. We prove by induction on $k$. Let $k=2$. Then, if $\Delta c_{j}=\sum \mu_{j_{1}, j_{2}} c_{j_{1}} \otimes c_{j_{2}}$ for $c_{j} \in B_{H_{*}}$, we have

$$
v_{i_{1}} \wedge v_{i_{2}} \otimes c_{j}=\sum(-1)^{\left|v_{i_{2}}\right|\left|c_{j_{1}}\right|} \mu_{j_{1}, j_{2}}\left(v_{i_{1}} \otimes c_{j_{1}}\right) \wedge\left(v_{i_{2}} \otimes c_{j_{2}}\right),
$$

and so $\varepsilon\left(i_{1}, j_{1} ; i_{2}, j_{2}\right)=\left|v_{i_{2}}\right|\left|c_{j_{1}}\right|$.

Let $k \geqslant 3$ and assume that the formula is true until $k-1$. Since $\Delta^{(k-1)}=$ $(\Delta \otimes \mathrm{id} \otimes \cdots \otimes \mathrm{id}) \circ \Delta^{(k-2)}$, if $\Delta^{(k-1)} c_{j}=\sum \mu_{j_{1}, \ldots, j_{k}} c_{j_{1}} \otimes \cdots \otimes c_{j_{k}}$ for $c_{j} \in B_{H_{*}}$, we can denote

$$
\Delta^{(k-2)} c_{j}=\sum \mu_{j_{1}^{\prime}, j_{3}, \ldots, j_{k}} c_{j_{1}^{\prime}} \otimes c_{j_{3}} \otimes \cdots \otimes c_{j_{k}}
$$


with $\Delta c_{j_{1}^{\prime}}=\sum \mu_{j_{1}, j_{2}}^{\prime} c_{j_{1}} \otimes c_{j_{2}}$ and $\mu_{j_{1}, \ldots, j_{k}}=\mu_{j_{1}, j_{2}}^{\prime} \mu_{j_{1}^{\prime}, j_{3}, \ldots, j_{k}}$. Then, by putting $v_{i_{1}^{\prime}}=v_{i_{1}} \wedge v_{i_{2}}$, we have

$$
\begin{aligned}
& v_{i_{1}} \wedge \cdots \wedge v_{i_{k}} \otimes c_{j} \\
& =v_{i_{1}^{\prime}} \wedge v_{i_{3}} \wedge \cdots \wedge v_{i_{k}} \otimes c_{j} \\
& =\sum(-1)^{\varepsilon\left(i_{1}^{\prime}, j_{1}^{\prime} ; i_{3}, j_{3} ; \ldots ; i_{k}, j_{k}\right)} \mu_{j_{1}^{\prime}, j_{3}, \ldots, j_{k}}\left(v_{i_{1}^{\prime}} \otimes c_{j_{1}^{\prime}}\right) \wedge\left(v_{i_{3}} \otimes c_{j_{3}}\right) \wedge \cdots \wedge\left(v_{i_{k}} \otimes c_{j_{k}}\right) .
\end{aligned}
$$

Furthermore, since

$$
v_{i_{1}^{\prime}} \otimes c_{j_{1}^{\prime}}=v_{i_{1}} \wedge v_{i_{2}} \otimes c_{j_{1}^{\prime}}=\sum(-1)^{\left|v_{i_{2}}\right|\left|c_{j_{1}}\right|} \mu_{j_{1}, j_{2}}^{\prime}\left(v_{i_{1}} \otimes c_{j_{1}}\right) \wedge\left(v_{i_{2}} \otimes c_{j_{2}}\right),
$$

we have

$$
\begin{aligned}
& v_{i_{1}} \wedge \cdots \wedge v_{i_{k}} \otimes c_{j} \\
& =\sum(-1)^{\varepsilon\left(i_{1}^{\prime}, j_{1}^{\prime} ; i_{3}, j_{3} ; \ldots ; i_{k}, j_{k}\right)+\left|v_{i_{2}}\right|\left|c_{j_{1}}\right|} \mu_{j_{1}, \ldots, j_{k}}\left(v_{i_{1}} \otimes c_{j_{1}}\right) \wedge \cdots \wedge\left(v_{i_{k}} \otimes c_{j_{k}}\right),
\end{aligned}
$$

and so

$$
\begin{aligned}
& \varepsilon\left(i_{1}, j_{1} ; \ldots ; i_{k}, j_{k}\right) \\
& =\varepsilon\left(i_{1}^{\prime}, j_{1}^{\prime} ; i_{3}, j_{3} ; \ldots ; i_{k}, j_{k}\right)+\left|v_{i_{2}}\right|\left|c_{j_{1}}\right| \\
& =\left(\left|v_{i_{3}}\right|+\cdots+\left|v_{i_{k}}\right|\right)\left|c_{j_{1}^{\prime}}\right|+\sum_{l=3}^{k-1}\left(\left|v_{i_{l+1}}\right|+\cdots+\left|v_{i_{k}}\right|\right)\left|c_{j_{l}}\right|+\left|v_{i_{2}}\right|\left|c_{j_{1}}\right| \\
& =\sum_{l=1}^{k-1}\left(\left|v_{i_{l+1}}\right|+\cdots+\left|v_{i_{k}}\right|\right)\left|c_{j_{l}}\right|
\end{aligned}
$$

because $\left|c_{j_{1}^{\prime}}\right|=\left|c_{j_{1}}\right|+\left|c_{j_{2}}\right|$.

Now we can prove Proposition 4.1.

Proof of Proposition 4.1. Notice that $\operatorname{cup}(X ; \mathbb{Q})<\infty$ since $X$ is finite-dimensional.

First, suppose that $\operatorname{dl}(Y)=\infty$. Then, since $d=0$, we have $\delta=\rho^{-1}(d \otimes \mathrm{id}) \rho=0$, and so $\bar{\delta}=0$.

Next, suppose that $\mathrm{dl}(Y)=m<\infty$. Fix a basis $B_{H_{*}}=\left\{c_{0}=1, c_{1}, c_{2}, \ldots\right\}$ for $H_{*}(X ; \mathbb{Q})$ with $0<\left|c_{1}\right| \leqslant\left|c_{2}\right| \leqslant \cdots$ and a basis $B_{V}=\left\{v_{1}, v_{2}, \ldots\right\}$ for $V$ with $0<\left|v_{1}\right| \leqslant\left|v_{2}\right| \leqslant \cdots$. Then, for an arbitrary element $v_{i} \in B_{V}$, we may denote

$$
d v_{i}=\sum_{k \geqslant m} \lambda_{i_{1}, \ldots, i_{k}} v_{i_{1}} \wedge \cdots \wedge v_{i_{k}}
$$

where $0 \neq \lambda_{i_{1}, \ldots, i_{k}} \in \mathbb{Q}$ and $v_{i_{1}}, \ldots, v_{i_{k}} \in B_{V}$ with $i_{1} \leqslant \cdots \leqslant i_{k}$.

(1). For an arbitrary element $c_{j} \in B_{H_{*}}$ with $c_{j} \neq 1$ and $k \geqslant m$, we may denote

$$
\Delta^{(k-1)} c_{j}=\sum \mu_{j_{1}, \ldots, j_{k}} c_{j_{1}} \otimes \cdots \otimes c_{j_{k}},
$$

where $0 \neq \mu_{j_{1}, \ldots, j_{k}} \in \mathbb{Q}$ and $c_{j_{1}}, \ldots, c_{j_{k}} \in B_{H_{*}}$. Since $\operatorname{cup}(X ; \mathbb{Q})<\operatorname{dl}(Y)=m$, by Lemma $4.2, \bar{\Delta}^{(k-1)} c_{j}=0$ for $k \geqslant m$, and so, by Lemma 4.3 , there exists an integer 
$s$ such that $c_{j_{s}}=1$ in each term of $\Delta^{(k-1)} c_{j}$ for $k \geqslant m$. Hence we have

$$
\begin{aligned}
& \delta\left(v_{i} \otimes c_{j}\right) \\
& =\sum_{k \geqslant m}(-1)^{\varepsilon\left(i_{1}, j_{1} ; \ldots ; i_{k}, j_{k}\right)} \lambda_{i_{1}, \ldots, i_{k}} \mu_{j_{1}, \ldots, j_{k}}\left(v_{i_{1}} \otimes c_{j_{1}}\right) \wedge \cdots \wedge\left(v_{i_{k}} \otimes c_{j_{k}}\right) \in J
\end{aligned}
$$

for an arbitrary element $v_{i} \in B_{V}$. Thus $\delta\left(\wedge\left(V \otimes H_{+}(X ; \mathbb{Q})\right) \in J\right.$ since $\delta$ is a derivation.

(2). Since $\operatorname{cup}(X ; \mathbb{Q}) \geqslant \operatorname{dl}(Y)=m$, by Lemma 4.2 , there exists an element $c_{j} \in B_{H_{*}}$ such that $c_{j} \neq 1$ and

$$
\bar{\Delta}^{(m-1)} c_{j}=\sum \mu_{j_{1}, \ldots, j_{m}} c_{j_{1}} \otimes \cdots \otimes c_{j_{m}} \neq 0 .
$$

Since $\operatorname{dl}(Y)=m$, there exists an element $v_{i} \in B_{V}$ such that $d v_{i}$ has a term of the form $\lambda_{i_{1}, \ldots, i_{m}} v_{i_{1}} \wedge \cdots \wedge v_{i_{m}}$ with $\lambda_{i_{1}, \ldots, i_{m}} \neq 0$ and $i_{1} \leqslant \cdots \leqslant i_{m}$. Then $\delta\left(v_{i} \otimes c_{j}\right)$ has terms of the form

$$
\sum(-1)^{\varepsilon\left(i_{1}, j_{1} ; \ldots ; i_{m}, j_{m}\right)} \lambda_{i_{1}, \ldots, i_{m}} \mu_{j_{1}, \ldots, j_{m}}\left(v_{i_{1}} \otimes c_{j_{1}}\right) \wedge \cdots \wedge\left(v_{i_{m}} \otimes c_{j_{m}}\right)
$$

with $c_{j_{s}} \neq 1$ for $1 \leqslant s \leqslant m$.

If $i_{1}<\cdots<i_{m}$, we see that each term $\left(v_{i_{1}} \otimes c_{j_{1}}\right) \wedge \cdots \wedge\left(v_{i_{m}} \otimes c_{j_{m}}\right)$ cannot be canceled by other terms.

If $i_{s}=i_{s+1}$ for some $s,\left|v_{i_{s}}\right|$ must be even. Then we have

$$
\begin{aligned}
& \left(v_{i_{s}} \otimes c_{j_{s}}\right) \wedge\left(v_{i_{s}} \otimes c_{j_{s+1}}\right) \\
& =(-1)^{\left(\left|v_{i_{s}}\right|-\left|c_{j_{s}}\right|\right)\left(\left|v_{i_{s}}\right|-\left|c_{j_{s+1}}\right|\right)}\left(v_{i_{s}} \otimes c_{j_{s+1}}\right) \wedge\left(v_{i_{s}} \otimes c_{j_{s}}\right) \\
& =(-1)^{\left|c_{j_{s}}\right|\left|c_{j_{s+1}}\right|}\left(v_{i_{s}} \otimes c_{j_{s+1}}\right) \wedge\left(v_{i_{s}} \otimes c_{j_{s}}\right)
\end{aligned}
$$

and, by Lemma 4.5 ,

$$
\begin{aligned}
\varepsilon & \left(i_{1}, j_{1} ; \ldots ; i_{s}, j_{s} ; i_{s}, j_{s+1} ; \ldots ; i_{m}, j_{m}\right) \\
& \quad-\varepsilon\left(i_{1}, j_{1} ; \ldots ; i_{s}, j_{s+1} ; i_{s}, j_{s} ; \ldots ; i_{m}, j_{m}\right) \\
= & \left(\left|v_{i_{s}}\right|+\left|v_{i_{s+2}}\right|+\cdots+\left|v_{i_{k}}\right|\right)\left|c_{j_{s}}\right|+\left(\left|v_{i_{s+2}}\right|+\cdots+\left|v_{i_{k}}\right|\right)\left|c_{j_{s+1}}\right| \\
& \quad-\left(\left|v_{i_{s}}\right|+\left|v_{i_{s+2}}\right|+\cdots+\left|v_{i_{k}}\right|\right)\left|c_{j_{s+1}}\right|-\left(\left|v_{i_{s+2}}\right|+\cdots+\left|v_{i_{k}}\right|\right)\left|c_{j_{s}}\right| \\
= & \left|v_{i_{s}}\right|\left(\left|c_{j_{s}}\right|-\left|c_{j_{s+1}}\right|\right) \equiv 0 \bmod 2 .
\end{aligned}
$$

Hence, by considering the coefficients with Lemma 4.4 , we see that each term $\left(v_{i_{1}} \otimes\right.$ $\left.c_{j_{1}}\right) \wedge \cdots \wedge\left(v_{i_{m}} \otimes c_{j_{m}}\right)$ cannot be canceled by other terms. (For example, see Example 3 in Section 5).

Thus there exists an element $v_{i} \otimes c_{j} \in \wedge\left(V \otimes H_{+}(X ; \mathbb{Q})\right)$ such that $\delta\left(v_{i} \otimes c_{j}\right) \notin$ $J$.

\section{Some examples}

Since $\operatorname{cup}(X ; \mathbb{Q})<\infty$ if $X$ is finite-dimensional and $\mathrm{dl}(Y)>1$ for any simply connected space $Y$ of finite type, we have

Proposition 5.1. Let $X$ be a formal, path connected, finite-dimensional CWcomplex of finite type and $Y$ a q-connected space of finite type with $q \geqslant \operatorname{dim} X$. 
Then, if $Y$ has a minimal Sullivan model of the form $(\wedge V, 0)$ or all cup products on $H^{+}(X ; \mathbb{Q})$ are trivial, $H^{*}\left(\mathcal{F}_{*}(X, Y) ; \mathbb{Q}\right)$ is always free.

Example 1. The following spaces have a minimal Sullivan model with a trivial differential:

- odd dimensional spheres,

- path connected $H$-spaces of finite type (cf. [3, Section 12(a), Example 3]),

- classifying spaces of path connected topological groups of finite type (cf. [3, Proposition 15.15]),

- Eilenberg-MacLane spaces of type $(\pi, n)$ with $n \geqslant 1, \pi$ is Abelian and $\pi \otimes_{\mathbb{Z}} \mathbb{Q}$ is finite dimensional (cf. [3, Section 15(b), Example 2]),

- a product of above spaces.

Example 2. The following spaces are formal and all cup products on the positive dimensional rational cohomology algebra are trivial:

- spheres,

- suspensions of spaces (cf. [3, Proposition 13.9]),

- co- $H$-spaces,

- a wedge of above spaces.

Note that a co- $H$-space is rationally homotopy equivalent to a wedge of spheres (cf. $[\mathbf{1}$, Section 7]), and a wedge of formal spaces is also formal.

A product of spheres $S^{i_{1}} \times \cdots \times S^{i_{n}}$ is an $\left(i_{1}+\cdots+i_{n}\right)$-dimensional CW-complex and a formal space with $\operatorname{cup}\left(S^{i_{1}} \times \cdots \times S^{i_{n}} ; \mathbb{Q}\right)=n$.

It is known that the $n$-th James reduced product space $J_{n}\left(S^{2 i}\right)$ of a $2 i$-dimensional sphere $S^{2 i}$ is a $2 n i$-dimensional $\mathrm{CW}$-complex which has the rational cohomology

$$
H^{*}\left(J_{n}\left(S^{2 i}\right) ; \mathbb{Q}\right)=\mathbb{Q}[c] /\left(c^{n+1}\right)
$$

with $|c|=2 i$, and has a minimal Sullivan model of the form

$$
\left(\wedge(v, \theta), d \theta=v^{n+1}\right)
$$

with $|v|=2 i$. Hence we have

Proposition 5.2. (1). Let $Y$ be a q-connected space of finite type with $q \geqslant i_{1}+$ $\cdots+i_{n}$. Then

$$
H^{*}\left(\mathcal{F}_{*}\left(S^{i_{1}} \times \cdots \times S^{i_{n}}, Y\right) ; \mathbb{Q}\right)
$$

is free if and only if $\mathrm{dl}(Y)>n$.

(2). Let $Y$ be a 2ni-connected space of finite type. Then

$$
H^{*}\left(\mathcal{F}_{*}\left(J_{n}\left(S^{2 i}\right), Y\right) ; \mathbb{Q}\right)
$$

is free if and only if $\mathrm{dl}(Y)>n$. 
(3). Let $X$ be a formal, path connected, p-dimensional $\mathrm{CW}$-complex of finite type with $p<2 i$. Then

$$
H^{*}\left(\mathcal{F}_{*}\left(X, J_{n}\left(S^{2 i}\right)\right) ; \mathbb{Q}\right)
$$

is free if and only if $\operatorname{cup}(X ; \mathbb{Q})<n+1$.

Example 3. $H^{*}\left(\mathcal{F}_{*}\left(S^{1} \times S^{3}, S^{6}\right) ; \mathbb{Q}\right)$ is not free.

Notice that $\operatorname{dl}\left(S^{6}\right)=2=\operatorname{cup}\left(S^{1} \times S^{3} ; \mathbb{Q}\right)$. A basis for $H_{*}(X ; \mathbb{Q})$ is given by $\left\{1, c_{1}, c_{3}, c_{4}\right\}$ with $\left|c_{j}\right|=j, \bar{\Delta} c_{1}=\bar{\Delta} c_{3}=0$ and

$$
\bar{\Delta} c_{4}=\mu_{1,3} c_{1} \otimes c_{3}+\mu_{3,1} c_{3} \otimes c_{1},
$$

where $\mu_{1,3}=(-1)^{1 \cdot 3} \mu_{3,1}=-\mu_{3,1}$. A minimal Sullivan model for $S^{6}$ is given by $\left(\wedge\left(v_{6}, v_{11}\right), d\right)$ with $\left|v_{i}\right|=i, d v_{6}=0$ and $d v_{11}=v_{6}^{2}$. By applying the construction described in Section 2, $\mathcal{F}_{*}\left(S^{1} \times S^{3}, S^{6}\right)$ has a minimal Sullivan model of the form

$$
\left(\wedge\left(\left\{v_{6}, v_{11}\right\} \otimes\left\{c_{1}, c_{3}, c_{4}\right\}\right), \bar{\delta}\right) .
$$

Then, by the formula (4.1) and Lemmas 4.4 and 4.5, we have

$$
\begin{aligned}
& \delta\left(v_{11} \otimes c_{4}\right) \\
& =(-1)^{6 \cdot 4}\left(v_{6} \otimes c_{4}\right) \wedge\left(v_{6} \otimes 1\right)+(-1)^{6 \cdot 0}\left(v_{6} \otimes 1\right) \wedge\left(v_{6} \otimes c_{4}\right) \\
& \quad+(-1)^{6 \cdot 1} \mu_{1,3}\left(v_{6} \otimes c_{1}\right) \wedge\left(v_{6} \otimes c_{3}\right)+(-1)^{6 \cdot 3} \mu_{3,1}\left(v_{6} \otimes c_{3}\right) \wedge\left(v_{6} \otimes c_{1}\right) \\
& =\left(v_{6} \otimes c_{4}\right) \wedge\left(v_{6} \otimes 1\right)+(-1)^{(6-0)(6-4)}\left(v_{6} \otimes c_{4}\right) \wedge\left(v_{6} \otimes 1\right) \\
& \quad+\mu_{1,3}\left(v_{6} \otimes c_{1}\right) \wedge\left(v_{6} \otimes c_{3}\right)+(-1)^{(6-3)(6-1)+1} \mu_{1,3}\left(v_{6} \otimes c_{1}\right) \wedge\left(v_{6} \otimes c_{3}\right) \\
& =2\left(v_{6} \otimes c_{4}\right) \wedge\left(v_{6} \otimes 1\right)+2 \mu_{1,3}\left(v_{6} \otimes c_{1}\right) \wedge\left(v_{6} \otimes c_{3}\right),
\end{aligned}
$$

and so $\bar{\delta}\left(v_{11} \otimes c_{4}\right)=2 \mu_{1,3}\left(v_{6} \otimes c_{1}\right) \wedge\left(v_{6} \otimes c_{3}\right) \neq 0$. 


\section{References}

[1] M. Arkowitz, Co-H-spaces, Handbook of algebraic topology, 1143-1173, North-Holland, 1995.

[2] E. H. Brown, JR. And R. H. Szczarba, On the rational homotopy type of function spaces, Trans. Amer. Math. Soc. 349 (1997), 4931-4951.

[3] Y. FÉLIX, S. Halperin And J.-C. Thomas, Rational homotopy theory, Graduate Texts in Mathematics 205, Springer-Verlag, 2001.

[4] K. KuribayAshi, Rational model for the evaluation map and iterated cyclic homology, preprint.

This article may be accessed via WWW at http://www.rmi.acnet.ge/hha/ or by anonymous ftp at

ftp://ftp.rmi.acnet.ge/pub/hha/volumes/2004/n1a18/v6n1a18.(dvi,ps,pdf)

Yasusuke KOTANI kotani@math.kochi-u.ac.jp

Department of Mathematics

Faculty of Science

Kochi University

Kochi 780-8520, Japan 